\title{
Assessoria Estatística e o Laboratório de Estatística Aplicada na Ensp-Fiocruz
}

\author{
Statistical Consulting and the Applied Statistical Laboratory \\ at Ensp-Fiocruz
}

\author{
Odécio Sanches ${ }^{1}$ \\ Cristina M. C. Amarante' \\ Takumi Iguchi ${ }^{1}$
}
SANCHES, O.; AMARANTE, C. M. C. \& IGUCHI, T. Statistical Consulting and the Applied Statistical Laboratory at Ensp-Fiocruz. Cad. Saúde Públ., Rio de Janeiro, 8 (1): 41-49, jan/mar, 1992.

The issue of statistical consulting in academic institutions to life sciences researchers is presented. In order to justify the importance of statistical methods in this area, a brief account of biostatistical ideas is made. Different types of statistical consulting are then presented, followed by the report of a current and local experience at the National School of Public Health, in Rio de Janeiro, Brazil. Finally, some recommendations are made.

Keywords: Biostatistics; Health Research; Statistical Consulting; Applied Statistics

\section{INTRODUÇÃO}

Na Escola Nacional de Saúde Pública (Ensp), a partir de maio de 1989 , por iniciativa de três membros componentes do Setor de Estatística do Departamento de Epidemiologia e Métodos Quantitativos em Saúde (DEMQS) e como parte de proposta de trabalho de professores visitantes, se inicia a instalação de um Laboratório de Estatística Aplicada (LEA), com múltiplos objetivos, a partir de atividades iniciais de assessoria estatística a pesquisadores da instituição, atividades estas que já vinham sendo desempenhadas de modo quase informal.

Em muitas instituições acadêmicas e/ou centros de pesquisa, as comissões constituídas para avaliação interna não consideram como relevantes as atividades de assessoramento estatístico.

O objetivo deste trabalho não é discutir os

\footnotetext{
'Departamento de Epidemiologia e Métodos Quantitativos em Saúde da Escola Nacional de Saúde Pública. Rua Leopoldo Bulhöes, 1480, Rio de Janeiro, RJ, 2104l, Brasil.
}

critérios de avaliação de tais comissões, o que não tem sentido como publicação, já que se trata de uma questão específica e interna a cada instituição. $O$ que pretendemos evidenciar, a partir dessa motivação, é o papel do assessoramento estatístico no controle de qualidade da análise de dados de pesquisa, em particular nas ciências da vida, e a posição dessa atividade diante daquelas de ensino, pesquisa e extensão de serviços, que são as atividades suportes de tais instituições.

\section{ESTATISTICA E BIOESTATÍSTICA: UM POUCO DE HISTÓRIA}

De modo sintético, pode-se registrar a Estatística como um conjunto de métodos e procedimentos para o tratamento, com fins descritivos e/ou inferencias, de um conjunto de dados onde a incerteza, inerente à variabilidade aleatória, se faz presente. Neste sentido, não é nenhuma heresia afirmar que a Estatística não produz conhecimento, mas é de fundamental importância a sua atuação na produção de conhecimento numa imensa variedade de áreas. 
Historicamente, operações de contagem de pessoas e avaliação de bens pessoais, isto é, atividades que hoje fazem parte de um censo populacional e econômico, eram já realizadas, de modo incipiente, no Egito do tempo dos faraós, na Grécia antiga e no Império Romano como atividades de interesse do Estado, seja com enfoque econômico associado à cobrança de taxas e impostos, seja para o controle da produção e distribuição de alimentos e/ou outros tipos de riquezas, seja por questões de segurança, no sentido de detectar quantos eram e onde estavam os potenciais inimigos representados pelos povos subjugados. Enfim, por alguma razão, sempre houve interesse em se conhecer o status populacional. Aí a origem da palavra Estatística.

Em Yule \& Kendall (1937) e em Berquó et al. (1980) podemos encontrar descrições detalhadas dos traços históricos, assim como um indicativo de textos onde aparecem, pela primeira vez, a palavra Estatística.

A partir do início deste século, a demanda por pesquisas com um maior embasamento científico-metodológico, principalmente nas áreas de genética, de agronomia e de antropologia, levou à criação de novos procedimentos de planejamento de investigação e de análise de dados na presença da variabilidade aleatória. Neste particular, surge com destaque o trabalho do matemático inglês Sir Ronald $\mathrm{A}$. Fisher, a partir da década de 20 , junto a pesquisadores em agronomia, na estação experimental de Rothanstead. Foi o verdadeiro trabalho de construção da Estatística, ao qual estão fortemente associados, também, os nomes de J. Neyman e E. S. Pearson. Também, na década de 30 , o matemático probabilista russo $A$. $N$. Kolmogorov formaliza a teoria moderna de probabilidades como uma teoria axiomática.

Todo este instrumental se consolida durante as décadas de 40 e 50 , colocando-se à disposição da investigação científica na área das chamadas soft sciences, onde a variabilidade aleatória é a característica dominante.

Problemas específicos nos vários campos da ciência passam a exigir procedimentos específicos de desenho e análise de dados.

Nas chamadas ciências da vida, se tem igual- mente, a partir da Segunda Guerra Mundial, o início de uma revolução científica. Fixemo-nos apenas em algumas poucas áreas, como, por exemplo, na Farmacologia, onde tivemos enorme progresso com a introdução de um conjunto de métodos estatísticos, denominados Ensaios Biológicos, para testes de potência de drogas e investigações do tipo dose-resposta, métodos estes calcados na teoria de regressão e na análise de variância. $\mathrm{Na}$ área dos Ensaios Clínicos Controlados, o trabalho pioneiro do inglês Sir Austin Bradford Hill sobre quantificação e aleatorização permitiu o desenvolvimento dos dois grandes ensaios de campo realizados, respectivamente, na Inglaterra, em 1946 , sobre o uso da estreptomicina no combate à tuberculose, e nos Estados Unidos, em 1952, sobre o efeito protetivo da gamaglobulina nos ataques da poliomielite. Paralelamente ao desenvolvimento dos Ensaios Clínicos Controlados, desenvolveu-se uma metodologia específica de Análise de Sobrevivência, aplicada aos ensaios clínicos terapêuticos, desde aqueles em pequena escala, nos laboratórios, até aos grandes ensaios multiclínicos. É também no desenvolvimento da moderna metodologia estatística que a Epidemiologia Teórica e Quantitativa vai encontrar os fundamentos para o seu desenvolvimento. Uma investigação científica mais rigorosamente fundamentada, na área das doenças transmissiveis, passa a exigir a criação de procedimentos específicos na área de processos estocásticos.

Como assinala Zelen (1985), a criação do National Institute for Health, na década de 40, nos Estados Unidos, pode ser considerada um exemplo da notável contribuição de elementos com forte background matemático e estatístico a áreas que envolvem os estudos de caso-controle, estudos de corte e estudo transversais; análise de dados discretos e dados categóricos; análise multivariada, ensaios biológicos e testes de segurança no uso de drogas, etc.

Conforme aponta Chiang (1985), a evolução das idéias no campo das ciências da vida levou à necessidade da criação de procedimentos estatísticos específicos. O conjunto desses procedimentos e métodos associados convencionou-se denominar Bioestatística. 
ASSESSORAMENTO ESTATÍSTICO: SUA POSIÇÃO FACE A OUTRAS ATIVIDADES ACADÉMICAS

$\mathrm{O}$ exposto no item anterior $\mathrm{e}$ um rápido exame de periódicos na área das ciências da vida nos dão uma idéia substancial da importância que é atribuída, e de fato possuída, aos procedimentos estatísticos nessa área.

Assim, apenas para exemplificar, tomando dois periódicos brasileiros na área de Saúde Pública - a Revista de Saúde Pública e os Cadernos de Saúde Pública - encontramos:

\section{a) Cadernos de Saúde Pública}

- Vol. VI, $\mathrm{n}^{2}$ 1, Janeiro/Março de 1990 - dos 8 artigos publicados, 6 utilizam algum tipo de procedimento estatístico;

- Vol. VI, $\mathrm{n}^{2} 2$, Abril/Junho de 1990 - dos 7 artigos publicados, 5 utilizam algum procedimento estatístico.

\section{b) Revista de Saúde Pública}

- Vol 24, n 3, Junho de 1990 - dos 9 artigos publicados, 8 utilizam procedimentos estatísticos;

- Vol. 24, $\mathrm{n}^{\mathrm{2}}$ 6, Dezembro de 1990 - dos 8 artigos publicados, 6 utilizam procedimentos estatísticos.

Queremos ressaltar, nn entanto, que o importante não é usar qualquer procedimento estatístico, mas sim, quando couber, usar o procedimento estatístico adequado.

Poucas universidades e/ou centros de pesquisa, entre nós, têm tido a preocupação de assegurar que seus esforços de pesquisa empreguem a Estatística de uma maneira apropriada. Especificamente, muitas dessas instituições não possuem qualquer estrutura formal que ofereça a possibilidade de assessoria estatística a seus pesquisadores.

A assessoria estatística deve representar um esforço para qualificar a pesquisa, melhorando os aspectos estatísticos da mesma no que diz respeito ao desenho (seja de experimentos, seja de surveys), à coleta, descrição, análise e interpretação dos dados. Ao mesmo tempo, dependendo da natureza do problema, a assessoria dá oportunidade ao estatístico de estabelecer idéias e propostas de novas metodologias e procedimentos.

A assessoria estatistica requer um considerável embasamento teórico, treinamento adequado e habilidades especificas. Conseqüentemente, exige que sua condução seja feita sob a supervisão de pessoal universitário altamente qualificado, em geral a nível de pós-graduação, e devidamente treinado nessa tarefa.

Como situar tal atividade nas instituições universitárias?

É universalmente reconhecido que a ordem de importância para as atividades no ambiente acadêmico são o ensino, a pesquisa e os serviços (estes sob a modalidade de extensão de serviços à comunidadade). Entre nós, também se dá importância, em um grau variável, às atividades burocrático-administrativas.

A assessoria estatística, em sua essência, não é ensino nem pesquisa e tem somente alguns aspectos daquilo que denominamos serviços. Estes fatores tornam a organização para o fornecimento da assessoria estatística, no ambiente acadêmico, uma questão que, às vezes, vem acompanhada de incompreensões $e$ aborrecimentos.

$O$ fato de a assessoria não ser uma atividade de ensino é particularmente embaraçoso, principalmente nas instituições acadèmicas mantidas pelo poder público, onde critérios de avaliação para efeito de distribuição de recursos, promoção e salário levam em consideração o número de disciplinas do departamento, o número de horas-aula, a relação discente/docente.

A atividade de assessoria estatística não é uma atividade de pesquisa para os estatísticos que estão nela engajados, uma vez que o produto desta atividade não gera, na quase totalidade das situaçōes, publicações na literatura estatística, raramente gerando publicações, em co-autoria, na área objeto da pesquisa assessorada.

Ainda que, a nível acadêmico, o desejável é que o docente também seja um pesquisador, de modo que o ensino possa se beneficiar da sua pesquisa, o fato de ensino não ser pesquisa é uma questão problema, no sentido em que a 
atividade de pesquisa é aquela que mais gera reconhecimento e, conseqüentemente, prestígio pessoal, principalmente pela divulgação que se dá ao nome do departamento e, portanto, ao nome da instituição, o que, como conseqüência, leva ao carreamento de recursos de fora para dentro (sejam recursos financeiros, materiais ou humanos).

Então, se a assessoria estatística deve ser reconhecida como uma atividade dentro da instituição, ela deve ser rotulada como um serviço ou, pelo menos, uma extensão de serviço. No entanto, esta é, por várias razões, uma classificação não satisfatória. De fato:

a) o aspecto serviço dá origem não só a problemas óbvios de reconhecimento profissional, mas também pode restringir a atração de fundos e recursos destinados à pesquisa;

b) o apecto serviço cria, entre os não-usuários, a sensação de um atendimento privilegiado, dentro de um escopo limitado, o que pode induzir, erroneamente, à conclusão de que esta é uma atividade de baixo consumo de tempo, que envolve um relacionamento interpessoal, se dando, pois, de forma errática, não responsavelmente credenciada, dirigida somente a uma parte dos membros da comunidade;

c) ainda sob o aspecto serviço, a atividade de assessoria estatística, no ambiente acadêmico, não apresenta nenhum paralelo com outras atividades.

A assessoria estatística adequada implica em um envolvimento muito forte do(s) assessor(es) e da equipe assessorada - em realidade, deve ser uma interação que se inicia na fase da definição dos objetivos e do planejamento propriamente dito, passando pelo monitoramento dos dados coletados, atingindo a fase de análise e culminando com a interpretação e conclusão, se a equipe assessorada deseja estar segura de que uma análise estatisticamente válida está sendo conduzida.

\section{POSSIVEIS ESTRUTURAS DE ASSESSORAMENTO ESTATISTICO A NÍVEL ACADÊMICO}

Considerando a experiência dos autores, vivenciada ao longo de muitos anos, em distintas instituições nacionais, tentar-se-á uma descrição entre váriás possíveis, de tipos de estruturas de assessoramento estatístico, seguindo uma linha apresentada por Gibbons \& Freund (1980).

\section{Assessoria Fornecida por Expert Não Estatístico}

Esta é, talvez, a situação mais comum em nossas instituições de ensino e pesquisa. Em um dado departamento, existe um (às vezes mais do que um) profissional da área que é considerado um especialista no uso de procedimentos estatísticos para a referida área: é o expert em estatística. Por exemplo, em um departamento de farmacologia, um farmacólogo devidamente treinado na metodologia dos Ensaios Biológicos passa a ser considerado, formal ou informalmente, um especialista em estatistica; em um departamento de medicina social, um pesquisador da área se especializa no uso da metodologia estatística aplicada a surveys sociais: passa a ser o expert em estatística no departamento.

O assessoramento estatístico fornecido via atuação de um expert é, na maioria dos casos, limitado e restrito aos conhecimentos estatisticos adquiridos, quase sempre, com enfoque centrado apenas nas necessidades da área. Isto tende não só a perpetuar o estreitamento do âmbito estatístico para a assessoria, mas também, em conseqüência, eliminar uma possível fonte de problemas desafiadores na sua natureza estatística.

\section{Assessoramento Embasado em Responsabilidade Determinada}

Esse tipo de assessoramento estatístico, raro entre nós, ocorre quando um departamento acadêmico é designado, ou aceita livremente, a incumbência e responsabilidade para a assessoria através de profissionais estatísticos titulados e devidamente treinados para tal.

Em tal tipo de estrutura organizacional, há uma tendência para a garantia de um maior espectro de qualidade no serviço de assessoria do que, possivelmente, seria oferecido por um expert.

Quase sempre, tal incumbência é assumida por um departamento de matemática ou de 
estatística, quando a instituição os possui. Em geral, tais tarefas são assumidas por profissionais estatísticos, alocados em algum departamento responsável pelo ensino dos vários programas e disciplinas de conteúdo programático em Estatística.

A assessoria estatística pode não ser a principal atividade do departamento, e se não for, o sistema terá grande dificuldade de ser realmente efetivo. A tarefa passa a ser de interesse secundário, tanto por parte dos assessores quanto do próprio departamento e da instituição como um todo, pondo em risco a qualidade da pesquisa, que depende de métodos estatísticos mais específicos e mais refinados, os quais geralmente não estão apresentados em livros textos.

Por outro lado, se o assessorado está convicto de que a atividade de assessoria é encarada, pelo profissional e pela instituição, como uma atividade de importância secundária, poderá preferir buscar assessoria através de um expert, ainda que tal atitude possa resultar em um tratamento estatístico talvez pobre ou inadequado.

\section{Assessoramento Estatistico Fornecido por Centros de Computação}

Entre as atividades de centros e/ou serviços de computaçāo está aquela de produzir programas ou pacotes de programas computacionais, segundo as necessidades da instituição e usuários, mantendo, simultaneamente, um serviço de assessoria para a utilização dos referidos programas. Desde que muitos desses programas são de natureza estatística, é inevitável que tais centros sejam procurados para fornecer assessoramento estatístico.

Programadores e/ou técnicos em computação são, em geral, estudantes não graduados ou, quando muito, com graduação em alguma área onde as atividades técnicas $\mathrm{e}$ as bases da programação são ensinadas.

Centros de computação, nestas condições, devem resistir à tentação de fornecer os serviços de assessoramento estatístico. Às vezes isto é difícil, quase impossível, jả que pesquisadores científicos quase sempre são personagens ccnsagradas na instituição, ou têm o respaldo de alguma chefia de departamento, enquanto os profissionais procurados quase sempre são apenas ocupantes de cargos técnicos, considerados pessoal de apoio, que, com uma eventual recusa, poderão criar, para si próprios, sérias dificuldades na relação de trabalho e obstáculos para sua promoção profissional, correndo o risco, até mesmo, de serem despedidos quando se deparam com chefias truculentas.

Se um profissional de estatística está devida e competentemente treinado na atividade de assessoria, ele pode ser vinculado, funcional e profissionalmente, a uin centro de computação ou, então, exercer tal atividade no departamento acadêmico encarregado do ensino da Estatística, ao mesmo tempo em que se liga, para o exercicio da assessoria, ao centro de computação. Esta situação não é diferente já que os centros de computação não são, em sua essência, de natureza acadêmica e, assim, torna-se difícil atrair um profissional titulado que irá exercer atividades em um centro técnico, sob a chefia e supervisão, às vezes, de um profíssional menos titulado academicamente e com interesses de atividades em áreas distintas daquelas da Estatística. Mais cedo ou mais tarde, choques e atritos serão inevitáveis, o que contribuirá para uma péssima qualidade de serviço.

Quando o assessor estatístico em um centro de computação não é um profissional estatístico devidamente treinado, ou quando é um profissional estatístico com interesse e treinamento centrados nos aspectos computacionais da estatística, isto pode levar a uma superenfatização de tais aspectos, em detrimento de outros talvez muito mais importantes, como, por exemplo, os aspectos do desenho, a formulação e os pressupostos teóricos de modelagem estatística, proposições teóricas de validade da metodólogia a ser empregada, questões de sensibilidade e especificidade dos instrumentos de medidas, identificação e correção de biases, etc.

Vale lembrar aqui que o uso indiscriminado de pacotes estatísticos, sem um exame mais aprofundado por parte de estatísticos profissionais, como acentua Iguchi (1990), é uma 
atitude que pode criar sérios embaraços para o pesquisador, pondo em risco a qualidade e adequação da análise estatística realizada.

\section{Consulta Estatística (?) do Tipo: "Hei?!.. Posso Falar com Você Só um Minutinho?"}

A interrogação entre parênteses significa nossa impotência para encontrar um qualificativo para nomear tal tipo de abordagem, que, entre nós, infelizmente, é um dos mais comuns.

É sério porque é desrespeitoso e reflete a ignorância total, por parte de quem o utiliza, em relação aquilo que está procurando.

Em geral isso ocorre quando alguém abre a porta de sua sala ou gabinete de trabalho, não pede licença, e quando você levanta a cabeça para verificar o que está ocorrendo, o "invasor" está de pé à sua frente (às vezes até já puxou uma cadeira e está comodamente sentado, encarando-o frente à frente) e, ao mesmo tempo, dizendo: "Você não me conhece, sou fulano(a) de tal, pertenço ao Departamento $X$, sou pesquisador(a) na área tal e quero um minutinho da sua atenção" (como se a unidade de tempo pudesse ser distribuída por diminutivos ou aumentativos!).

Antes que você consiga sair da sua perplexidade (nós agora já não ficamos muito perplexos) e articular alguma coisa do tipo "Vocé poderia vir mais tarde, agora estou ocupado", ou coisa equivalente, o "invasor" já respirou o suficiente para disparar: "Sabe? Terei que entregar minha tese amanhã e preciso resolver só umas "questõezinhas" (todas, às vezes, até remendar introduçāo, objetivos, material e métodos, etc) e quero que você me ajude. Caso contrário, terei sérios problemas com a Comissão de Pós-Graduação". Ou, então, a colocação é "Sabe? Eu mandei este artigo para a Revista $Z$ e mandaram de volta com algumas recomendaçōes sobre o uso de estatística. Como eu não entendo nada disto, quero que você faça para mim".

$E$ incrivel que isto possa ocorrer a nível acadêmico, mas ocorre e reflete, além da ignorância já referida, uma enorme desconsideração às atividades profissionais.

\section{ASSESSORIA ESTATISTICA NA ENSP VIA LABORATÚRIO DE ESTATÍSTICA APLICADA}

Em instituições acadêmicas e/ou centros de pesquisa de grande porte que desenvolvem simultaneamente atividades de ensino e pesquisa, o serviço de assessoramento estatístico pode ser fornecido através de uma unidade específica, que poderá ser mais ou menos complexa em função do porte da instituição.

Laboratório de Estatística Aplicada (LEA) é um rótulo, nas universidades que dispõem de cursos de formação de profissionais estatísticos (Bacharelado em Estatística), para designar um ambiente físico onde são desenvolvidas as atividades de treinamento de alunos de graduação e pós-graduação em assessoramento estatístico. Assim, na Faculdade de Medicina de Ribeirão Preto, da USP, onde não havia graduação em Estatística, mas um Mestrado em Bioestatística, o serviço de assessoramento estatístico associado ao treinamento de pós-graduandos era rotulado "Ambulatório de Estatística".

Na Escola Nacional de Saúde Pública, até o primeiro semestre de 1989, apesar da presença de estatísticos, as atividades de assessoria estatística eram exercidas com base na estrutura tipo expert e, às vezes, informalmente, na base do "Hei?! posso falar com você um minutinho?".

O Departamento de Epidemiologia e Métodos Quantitativos em Saúde (DEMQS), antes desta data, a fim de atender suas necessidades de ensino e pesquisa, particularmente no ensino que envolve residência médica, especialização, mestrado (em duas áreas) e doutorado (em três áreas temáticas), já havia proposto à Direção da Instituição a criação e instalação do "Setor de Análise de Dados e Computação". Em setembro de 1989 , foi anexado à referida solicitação um adendo sobre a criação e instalação de um Laboratório de Estatística, o que foi encaminhado e aprovado pelos órgãos dirigentes da Ensp.

A justificativa para a criação e instalação de tal laboratório era a seguinte:

O Laboratório de Estatística deve ter um 
ambiente físico dotado de recursos humanos e materiais indispensáveis para o bom desenvolvimento e aplicação de métodos estatísticos $\mathrm{e}$ programas computacionais em estatística, objetivando o adequado planejamento e análise de dados nas várias áreas da pesquisa em Saúde Pública.

Estabelecemos três vertentes, em relação às quais se percebe o papel fundamental do Laboratório e seus recursos computacionais:

I. Dentro das funções de desenvolvimento e aplicação de métodos estatísticos no planejamento das investigações e na análise de dados, o Laboratório funciona como um setor de extensão de serviços à comunidade científica da Fundação Oswaldo Cruz (Fiocruz).

Os pesquisadores são atendidos em um sistema de assessoria, numa escala hierárquica de prioridades que vai dos Docentes/Pesquisadores do DEMQS, passando àqueles dos demais Departamentos da Ensp, para atingir os das demais Unidades da Fiocruz, podendo se estender a outros órgãos do Ministério da Saúde, quando solicitado.

Estas atividades já vêm sendo desenvolvidas desde maio de 1989 , ainda que o setor não conte com uma infra-estrutura adequada de equipamentos computacionais nem com uma infra-estrutura completa de recursos humanos de apoio técnico.

II. Uma outra vertente está associada ao desenvolvimento dos recursos humanos que compōem o Laboratório de Estatística. Isto pode ser compreendido como essencial e inerente ao processo de trabalho da equipe que compõe o Laboratório, uma vez que as solicitações de assessoria envolvem, muitas vezes, novas questōes metodológicas que, por sua vez, irão levar à criação ou adaptação de novas técnicas e procedimentos.

III. Uma terceira vertente diz respeito ao atendimento e/ou treinamento de alunos de pós-graduação. $\mathrm{O}$ atendimento de pós-graduandos, no desenho e análise de dados de suas dissertações e/ou teses, se fará nos mesmos moldes do assessoramento estatístico a pesquisadores/docentes. $O$ treinamento se fará por um processo de orientação intensiva quando o pós-graduando tiver necessidade de um maior aprofundamento na metodologia estatistica.
Este projeto foi aprovado pelo Conselho Deliberativo da Ensp durante o ano de 1990 e, ao final desse ano, iniciaram-se as obras (ainda em andamento para a adaptação do ambiente físico).

Como se percebe pelo exposto, o nome Laboratório de Estatística é, também aqui, um rótulo que poderia ter sido substituído por qualquer outro do tipo: Setor de Assessoramento Estatístico, Setor de Apoio Estatístico à Pesquisa, etc.

Desde maio de 1989, quando se iniciaram, de modo formal e controlado por sistema de registro de inscrições e acompanhamento, as atividades do Laboratório de Estatística, estas vêm sendo desempenhadas por uma equipe constituída de três Docentes/Pesquisadores com formação pós-graduada em Estatística, dois dos quais são visitantes dentro de Convênio CNPq-Fiocruz.

Apesar da insuficiência de recursos humanos especializados e de recursos humanos de apoio técnico, as atividades se desenvolvem ininterruptamente, com reuniōes semanais $\mathrm{e}$ dois atendimentos por reunião.

Os dados a seguir resumem estas atividades de assessoramento estatístico até o presente momento:

TABELA 1. Atendimento do Laboratório de Estatistica Aplicada - DEMQS/ Ensp/Fiocruz, segundo Instituição de Origem, no Período de Funcionamento: Maid/89 a Maio/91

\begin{tabular}{lcccc}
\hline \hline $\begin{array}{c}\text { Instihuçáo } \\
\text { de } \\
\text { Origem }\end{array}$ & 1989 & 1990 & 1991 & Tota! \\
\cline { 2 - 5 } & & & \\
\hline DEMQS & 4 & 6 & 3 & 13 \\
Ensp $^{*}$ & - & 4 & 1 & 5 \\
Fincriz $^{*}$ & 2 & 3 & 4 & 0 \\
Outros & 4 & 2 & - & 6 \\
\hline Total & 10 & 15 & 8 & 33 \\
\hline \hline
\end{tabular}

(1) Excluido o iten anterior

\section{CONCLUSÃO E RECOMENDAÇÕES}

Considerando o exposto e as experiencias desenvolvidas, seja esta em andamento na Ensp, sc jam outras em distinlas instituições do 
país, concluimos que, na Ensp, ainda que com dificuldades e com deficiências em virtude da estrutura incompleta, as atividades de assessoramento estatístico mostram-se necessárias e têm sido desenvolvidas a contento, do nosso ponto de vista.

Além do mais, o que foi exposto permite estabelecer as seguintes recomendações:

a) Os serviços de assessoramento estatístico, em qualquer instituição, devem ser complementados pelo acesso às facilidades de computação;

b) Idealmente, deverá ser constituído um pool de assessores de modo a agregar profissionais estatísticos de diferentes especialidades, principalmente em função do porte da instituição e da demanda dos serviços pelas mais diversas áreas do conhecimento;

c) Estudantes de graduação, da residência e pós-graduandos (lato sensu e stricto sensu) podem e devem ser inseridos na estrutura; d) Sempre que possivel, a estrutura de expert deverá ser substituída por uma estrutura de responsabilidade determinada, devendo-se evitar o assessoramento por técnicos de computação e erradicar a estrutura do tipo "Hei?! você pode me dar um minutinho?";

e) Em função do porte da instituição e da demanda por assessoria estatística, os profissionais estatísticos engajados nessa atividade devem tê-la como tarefa única, isto é, deve ser criada uma unidade ou setor com a finalidade exclusiva de assessoramento estatístico;

f) Independentemente da situação anterior, as instituições devem considerar, para efeito de avaliação, promoções, gratificações e aumentos de salários, as atividades de assessoramento estatístico no mesno grau de importância das demais atividades (de ensino, pesquisa cientifica, participação na administração e/ou colegiados).

TABELA 2. Atendimento do Laboratório de Estatistica Aplicada - DEMQS/Ensp/Fiocruz, segundo Áreas de Concentração, no Periodo de Funcionamento: Maid89 a Maio/91

\begin{tabular}{lcccc}
\hline \hline \multirow{1}{*}{$\begin{array}{c}\text { Área } \\
\text { de } \\
\text { Concentração }\end{array}$} & 1989 & 1990 & 1991 & Total \\
\cline { 2 - 4 } & 6 & 7 & 3 & 16 \\
\hline Epidemiologia & 3 & 4 & 2 & 9 \\
Vacina/Imunologia & - & 3 & 1 & 4 \\
$\begin{array}{l}\text { Planejamentod } \\
\text { Administração em Saúde }\end{array}$ & 1 & 1 & 2 & 4 \\
Ecologia/ & 10 & 15 & 8 & 33 \\
Biologia Geral & & & & \\
\hline Total &
\end{tabular}

TABELA 3. Atendimento do Laboratório de Estatística Aplicada - DEMQS/Ensp/Fiocruz, segundo a Finalidade da Pesquisa Assessorada, no Período de Funcionamento: Maid1989 a Maid1991

\begin{tabular}{lcccc}
\hline \hline \multirow{2}{*}{$\begin{array}{c}\text { Finalidade } \\
\text { da } \\
\text { Pesquisa }\end{array}$} & 1989 & 1990 & 1991 & Total \\
\cline { 2 - 4 } & 6 & 6 & 4 & 16 \\
\hline Publicação & 3 & 5 & 3 & 11 \\
Dissertação de Mestrado & 1 & 4 & 1 & 6 \\
Tese de Doutorado & 10 & 15 & 8 & 33 \\
\hline Total & & & & \\
\hline \hline
\end{tabular}




\section{AGRADECIMENTOS}

Ao Convênio Fiocruz-CNPq, que permitiu a realização deste trabalho com a concessão das bolsas de Professor-Visitante.

Ao referee pelas sugestões.

À Sra. Leila Rasina Mendes pela dedicação na elaboração do texto computadorizado.

\section{RESUMO}

SANCHES, O; AMARANTE, C. M. C. \& IGUCHI, T. Assessoria Estatística e o Laboratório de Estatística Aplicada na Ensp-Fiocruz. Cad. Saúde Públ., Rio de Janeiro, 8 (1): 41-49, jan/abr, 1992.

Baseados na experiência vivenciada em várias instituições e, em particular, numa experiência em andamento, os autores discutem a questão do assessoramento estatístico a pesquisadores nas áreas que compõem as ciências da vida. Fazendo um breve comentário da evolução dos métodos e procedimentos estatísticos aplicados às ciências da vida, justificam a consolidação da Bioestatística como área especifica. Discutem as vantagens e desvantagens de vários tipos de estruturas de assessoramento estatístico que, obviamente, podem ser estendidas a outros tipos de assessoramento. Apresentam a proposta de uma experiência, em andamento, de criação de um Laboratório de Estatística Aplicada na Ensp (Escola Nacional de Saúde Pública) e terminam por apresentar algumas recomendaçōes.

Palavras-Chave: Bioestatística; Pesquisa em Saúde; Assessoramento Estatístico; Estatística Aplicada

\section{REFERÊNCIAS BIBLIOGRÁFICAS}

BERQUÓ, E. S.; SOUZA, J. M. P. de \& GOTLIEB, S. L. D., 1980. Bioestatistica, São Paulo: E.P.U.. CHIANG, C. L., 1985. Reader Reaction: What is Bioestatistics? Biometrics, 41: 771-773.

GIBBONS, J. D. \& FREUND, R. J., 1980. Organizations for statistical consulting at colleges and universities. American Statistician, 34: 140-145.
IGUCHI, T., 1990. Fugindo da catástrofe. Em Dia com a Ensp, 1 (7): 5.

ZELEN, M., 1985. Response. Biometrics, 41: 773-775.

YULE, G. U. \& KENDALL, M. C., 1937. An Introduction to the Theory of Statistics. London: Charles Griffin \& Co. 